\title{
Ultra-processed food consumption and excess weight among US adults
}

\author{
Filippa Juul $^{1}$, Euridice Martinez-Steele ${ }^{2,3}$, Niyati Parekh ${ }^{1,4}$, Carlos A. Monteiro ${ }^{2,3}$ and Virginia W. Chang ${ }^{1,4 *}$ \\ ${ }^{1}$ College of Global Public Health, New York University, New York, NY 10012, USA \\ ${ }^{2}$ School of Public Health, University of São Paulo, São Paulo, 03178-200, Brazil \\ ${ }^{3}$ Center for Epidemiological Studies in Health and Nutrition, University of São Paulo, São Paulo, 03178-200, Brazil \\ ${ }^{4}$ School of Medicine, New York University, New York, NY 10016, USA \\ (Submitted 17 November 2017 - Final revision received 17 March 2018 - Accepted 24 March 2018 - First published online 6 May 2018)
}

\section{Abstract}

Ultra-processed foods provide $58 \%$ of energy intake and $89 \%$ of added sugars in the American diet. Nevertheless, the association between ultraprocessed foods and excess weight has not been investigated in a US sample. The present investigation therefore aims to examine the association between ultra-processed foods and excess weight in a nationally representative sample of US adults. We performed a cross-sectional analysis of anthropometric and dietary data from 15977 adults (20-64 years) participating in the National Health and Nutrition Examination Survey 20052014. Dietary data were collected by 24-h recall. Height, weight and waist circumference (WC) were measured. Foods were classified as ultraprocessed/non-ultra-processed according to the NOVA classification. Multivariable linear and logistic regression was used to evaluate the association between ultra-processed food consumption (\% energy) and BMI, WC and odds of BMI $\geq 25 \mathrm{~kg} / \mathrm{m}^{2}, \mathrm{BMI} \geq 30 \mathrm{~kg} / \mathrm{m}^{2}$ and abdominal obesity (men: WC $\geq 102 \mathrm{~cm}$, women: WC $\geq 88 \mathrm{~cm}$ ). Prevalence of $\mathrm{BMI} \geq 25 \mathrm{~kg} / \mathrm{m}^{2}, \mathrm{BMI} \geq 30 \mathrm{~kg} / \mathrm{m}^{2}$ and abdominal obesity was $69 \cdot 2,36 \cdot 1$ and $53.0 \%$, respectively. Consuming $\geq 74.2 v$. $\leq 36.5 \%$ of total energy from ultra-processed foods was associated with 1.61 units higher BMI $(95 \%$ CI $1 \cdot 11,2 \cdot 10), 4 \cdot 07 \mathrm{~cm}$ greater WC $(95 \%$ CI $2 \cdot 94,5 \cdot 19)$ and 48,53 and $62 \%$ higher odds of BMI $\geq 25 \mathrm{~kg} / \mathrm{m}^{2}, \mathrm{BMI} \geq 30 \mathrm{~kg} / \mathrm{m}^{2}$ and abdominal obesity, respectively (OR 1.48; $95 \%$ CI 1.25, 1.76; OR 1.53; $95 \%$ CI 1.29, 1·81; OR 1.62; $95 \%$ CI 1.39, 1.89, respectively; $P_{\text {for trend }}<0 \cdot 001$ for all). A significant interaction between being female and ultra-processed food consumption was found for BMI $\left(F_{4,79}=4 \cdot 89, P=0 \cdot 002\right), \mathrm{WC}\left(F_{4,79}=3 \cdot 71, P=0 \cdot 008\right)$ and BMI $\geq 25 \mathrm{~kg} / \mathrm{m}^{2}\left(F_{4,79}=5 \cdot 35, P<0 \cdot 001\right)$. As the first study in a US population, our findings support that higher consumption of ultra-processed food is associated with excess weight, and that the association is more pronounced among women.

Key words: Ultra-processed foods: Food processing: BMI: Overweight: Obesity: Waist circumference: National Health and Nutrition Examination Survey

Ultra-processed foods are defined as industrially manufactured, ready-to-eat or ready-to-heat formulations made mostly from substances that are derived from foods with little, if any, whole foods ${ }^{(1)}$. These products are typically convenient, omnipresent and sophisticatedly marketed; high in added sugars, salt and/or SFA; and contain cosmetic additives such as flavourings, colouring agents, emulsifiers and preservatives ${ }^{(1-4)}$. Ultraprocessed foods include, but are not limited to, so-called 'junk foods' such as soft drinks, potato chips and confectionery, and industrially manufactured baked goods ${ }^{(1)}$. However, several products that are often marketed and perceived as healthy, for example, granola bars, low-fat flavoured yogurt, low-energy frozen dinners, most industrially produced breads and canned soups, are also ultra-processed ${ }^{(1)}$.

Ultra-processed foods provide on average $58 \%$ of daily energy and $89 \%$ of added sugars in the US diet ${ }^{(5)}$. The nutritional value of ultra-processed foods and their role in a healthy diet is controversial. Although these foods may contribute to the intake of several essential nutrients in the American diet, they are also more likely to be excessive in SFA, added sugar and $\mathrm{Na}$ compared with less processed foods ${ }^{(4,6,7)}$. In addition, studies in the USA, UK, Canada, Chile, France and Brazil have consistently found diets with a higher proportion of ultra-processed foods to be of poorer nutritional quality ${ }^{(8-14)}$.

The recent abundance of ultra-processed food in the global food supply has been hypothesised as a contributor to the worldwide rise in overweight and obesity ${ }^{(2,15)}$. However, few epidemiological studies have examined ultra-processed foods as a group in relation to excess weight ${ }^{(10,12,16-18)}$. Greater household purchases of ultra-processed foods and consuming a higher proportion of daily energy from ultra-processed foods was associated with higher BMI and prevalence of overweight and obesity in two cross-sectional studies using nationally representative data from Brazil in 2008-2009 ${ }^{(16,18)}$. Similarly, higher consumption of ultra-processed foods was associated with higher prevalence of overweight and obesity in a cross-sectional study of over 74000 French adults ${ }^{(12)}$. In the prospective Seguimiento University of Navarra (SUN) study of

Abbreviations: NHANES, National Health and Nutrition Examination Survey; WC, waist circumference.

* Corresponding author: V. W. Chang, fax +1 212995 4877, email vc43@nyu.edu 
middle-aged Spanish college graduates, higher consumption of ultra-processed food was associated with significantly higher risk of developing overweight or obesity over approximately 9 years of follow-up ${ }^{(17)}$. Conversely, no association was observed between relative energy intake from ultra-processed and moderately processed foods combined and body weight in a crosssectional study of a nationally representative sample in the $\mathrm{UK}^{(10)}$.

Studies in the USA on specific ultra-processed foods have shown that fast food, potato chips, French fries, sugar-sweetened beverages, processed meat products, fried foods, sweets and desserts are associated with weight gain ${ }^{(19,20)}$. Nevertheless, the association between all ultra-processed foods as a group, that is not only specific 'junk' and fast foods, and excess weight has not been examined in a US population. Examining ultra-processed foods as a group, rather than just evaluating individual foods, is essential to our understanding of how food processing per se can affect health, body weight and adiposity, and this, in turn, can inform food-based dietary guidelines, weight management strategies and food and nutrition policies in the USA.

Given that ultra-processed foods provide the majority of energy in the average American diet ${ }^{(5)}$, it is of particular relevance to increase our understanding regarding the relationship between these foods as a group and excess weight in the USA. Importantly, findings from existing international studies may not generalise to the US setting owing to considerable national differences in food choices, dietary habits and population characteristics. Processing methods, ingredients and the types of ultra-processed foods that are available to consumers may also differ between countries. The objective of the present investigation is therefore to examine the association between consumption of ultra-processed foods and excess weight in a nationally representative sample of US adults.

\section{Methods}

\section{Data source}

We used the National Health and Nutrition Examination Survey (NHANES) survey cycles 2005-2006 through 2013-2014. NHANES is a cross-sectional, nationally representative survey of the US non-institutionalised civilian population ${ }^{(21)}$. Samples for each cycle were obtained using a complex, stratified, multistage probability cluster sampling design ${ }^{(21)}$.

\section{Data collection}

Data on demographics, socio-economic characteristics and health behaviours were self-reported during an interview by trained interviewers in the respondents' homes. Trained health technologists measured participants' height, weight and waist circumference (WC) during a health examination performed at a mobile examination centre ${ }^{(21-25)}$. Dietary intake data were collected through two 24-h dietary recall interviews ${ }^{(21)}$, using the validated US Department of Agriculture Automated Multiple-Pass Method (AMPM) ${ }^{(26-28)}$. The first recall was performed in-person at the time of the health examination, and the second recall was carried out over the telephone 3-10 d later. A total of 50965 individuals responded to the household interview, of whom 49116 individuals participated in the health examination $^{(29)}$. A total of 45538 individuals provided at least one valid 24-h dietary recall.

\section{Definition of exposure variable}

We used consumption of ultra-processed food (measured as percentage of total energy intake) categorised into quintiles as the exposure variable. To calculate this measure, we classified all food items recorded in NHANES 2005-2014 as ultraprocessed or non-ultra-processed on the basis of the extent and purpose of the industrial food processing applied in accordance with the NOVA food classification ${ }^{(1)}$. The scientific basis and logic of NOVA has been explained elsewhere ${ }^{(15,30,31)}$. NOVA defines ultra-processed foods as industrial formulations made with no or minimal whole foods and produced with substances extracted from foods or synthesised in laboratories such as dyes, flavourings and preservatives, often using processing techniques with no domestic equivalent such as extrusion or moulding ${ }^{(1)}$. The purpose of ultra-processing is to create convenient and intensely palatable products that are ready-to-drink, ready-to-eat or ready-to-heat and have a long shelf-life ${ }^{(1)}$. The following foods were considered to be ultra-processed: instant and canned soups; reconstituted meat and fish products; readymade sauces, gravies and dressings; French fries and other pre-made potato products such as chips; ready-to-eat and dry-mix desserts such as pudding; confectionery; sweet and savoury snack foods including granola bars and protein bars; sugar-sweetened or artificially sweetened beverages including soda, fruit drinks, pre-sweetened tea and coffee, energy drinks and dairy-based drinks; flavoured and/or sweetened yoghurt; industrially manufactured cakes, cookies and pies; dry cake and pancake mixes; industrially manufactured breads; sweet breakfast cereals; frozen and shelf-stable plate meals; ice cream, frozen yogurt and ice pops; meatless patties and fish sticks and infant formula ${ }^{(1)}$. Non-ultra-processed foods encompass 'unprocessed or minimally processed foods' including fresh, dry or frozen fruits or vegetables, grains, legumes, meat, fish and milk; 'processed culinary ingredients' including table sugar, oils, fats, salt and other constituents extracted from foods or from nature and used in kitchens to make culinary preparations; and 'processed foods' including foods such as canned fish and vegetables and artisanal cheeses, which are manufactured by adding salt, sugar, oil or other processed culinary ingredients to unprocessed or minimally processed foods ${ }^{(1)}$.

We classified foods by taking into account the NHANES variables 'Main Food Description', 'Additional Food Description', which describes foods (food codes), and 'SR Code Description', which describes the underlying ingredients of foods (SR codes). We also considered the NHANES variables 'Combination Food Type' and 'Source of Food'. In consequence, most foods categorised as 'Lunchables' or 'Frozen meals', or sourced from a 'Restaurant fast food/pizza' or 'Vending machine', were classified as ultra-processed. When foods were judged to be a hand-made recipe, we applied the classification to the underlying ingredients in order to ensure a more accurate classification. Two authors independently reviewed the classification of each item. Discrepant classifications were resolved by discussion. Fig. 1 describes the classification process. 


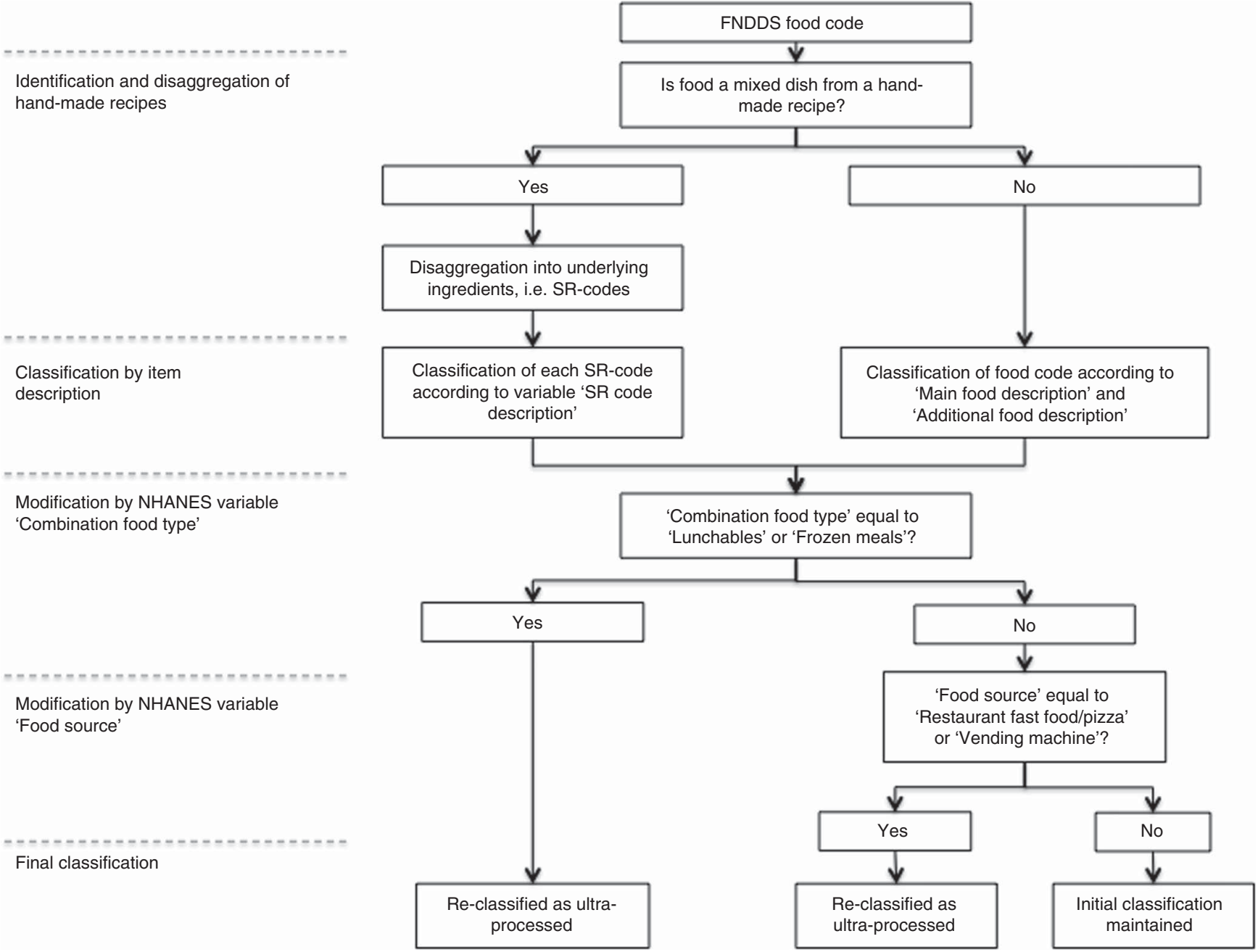

Fig. 1. Flow chart of the food classification process. FNDDS, Food and Nutrient Database for Dietary Studies; NHANES, National Health and Nutrition Examination Survey.

SR codes for each cycle were obtained from the corresponding versions of the US Department of Agriculture (USDA) Food and Nutrient Database for Dietary Studies (FNDDS) - that is FNDDS 3.0, 4.0, 5·0, 2011-2012 and 2013-2014. Food Code energy values provided by NHANES were used to calculate energy intakes from each NOVA food group. For hand-made recipes, energy values of the underlying ingredients (SR Codes) were calculated using data from both FNDDS and the USDA National Nutrient Database for Standard Reference, Release $20-28$. The procedures and calculations have been described in detail elsewhere ${ }^{(5)}$.

Using dietary data for day 1 , we calculated the relative contribution of ultra-processed products to total energy intake for each observation (\% of total energy). We thereafter divided the sample into quintiles according to relative dietary contribution of ultra-processed foods.

\section{Definition of outcome variables}

BMI $\left(\mathrm{kg} / \mathrm{m}^{2}\right)$ and WC $(\mathrm{cm})$ were used as measures of excess weight and abdominal obesity, respectively. BMI values of $\geq 25$ and $\geq 30 \mathrm{~kg} / \mathrm{m}^{2}$ were classified as 'overweight and obesity' and 'obesity', respectively, according to World Health Organization criteria $^{(32)}$. Abdominal obesity was defined as a WC $\geq 102 \mathrm{~cm}$ for men and $\geq 88 \mathrm{~cm}$ for women ${ }^{(33)}$.

\section{Definition of covariates}

Demographic covariates of interest included race/ethnicity (non-Hispanic white, non-Hispanic Black, Hispanic, other race including multi-racial), sex, age (in years, continuous) and marital status (married, divorced/widowed/separated, never married). Socio-economic status was measured by education level (less than high school, high school degree/general equivalency diploma, some college, college graduate or above) and family poverty income ratio, defined as the ratio of family income:the federal poverty threshold (continuous). Physical activity was classified as low $(<150 \mathrm{~min}$ of moderate intensity equivalent activity per week), medium (150-300 min of moderate intensity equivalent activity per week) and high (>300 min of moderate intensity equivalent activity per week), based on the fulfilment of the 2008 Physical Activity Guidelines 
for Americans ${ }^{(34)}$. Smoking status was categorised as never smoked, former smoker and current smoker.

\section{Inclusion and exclusion criteria}

The analytical sample was restricted to adults aged 20-64 years, as the association between BMI and adiposity may differ for senior compared with young and middle-aged adults. Individuals were included in the analyses if they had at least one valid dietary recall and complete data for BMI, WC and demographic, socio-economic and behavioural covariates. A total of 21637 participants were in the appropriate age span and eligible to be included in the analyses. Of these, we excluded pregnant and lactating women ( $n$ 455); participants who were underweight $\left(\mathrm{BMI}<18.5 \mathrm{~kg} / \mathrm{m}^{2} ; n\right.$ 355) or reported implausible energy intakes, defined as $<2092$ or $>14644 \mathrm{~kJ}$ $(<500$ or $>3500 \mathrm{kcal})$ for women and $<2510$ or $>18828 \mathrm{~kJ}$ ( $<600$ or $>4500 \mathrm{kcal}$ ) for men ( $n$ 899); and individuals missing data for outcomes, exposure or covariates ( $n$ 3951). Thus, the final sample of this study was 15977 participants.

\section{Statistical analyses}

We first assessed the distribution of participant demographic, socio-economic, dietary and behavioural characteristics by quintile of ultra-processed food consumption. Differences in individual and dietary characteristics by quintile of ultraprocessed food consumption were evaluated by Pearson's $\chi^{2}$ test of independence (categorical variables) and linear regression (continuous variables).

Multiple linear regression analysis was used to evaluate associations between relative energy intake of ultra-processed food and BMI and WC, respectively. Multiple logistic regression analysis was used to determine associations between relative energy intake of ultra-processed food and the odds overweight/ obesity, obesity and abdominal obesity, respectively. In both linear and logistic models, quintile of relative contribution of ultra-processed foods to total energy intake was included as the explanatory variable, with the first quintile as the reference category. For all outcomes, we first fitted an age-adjusted model and thereafter a multivariable model adjusted for age, sex, educational attainment, race/ethnicity, ratio of family income to poverty, marital status, smoking and physical activity level. Test of linear trend was performed by treating quintile of ultraprocessed food as an ordinal variable. On the basis of the multivariable regression models, we calculated and plotted the estimated margins for each outcome (BMI, WC and probability of overweight, obesity and abdominal obesity) according to quintile of relative ultra-processed food consumption. Margins were estimated at the means of all covariates.

All analyses were performed both for the full analytical sample and stratified by sex. We tested for interaction between relative consumption of ultra-processed food and sex, age (years, continuous) and age group (20-39, 40-59 and >59 years), respectively, by including an interaction term (female $\times$ quintile of ultra-processed food, age $\times$ quintile of ultra-processed food and age-group $\times$ quintile of ultra-processed food, respectively) in the multivariable models. As energy intake is a plausible mediator of the association between ultra-processed food consumption and excess weight and abdominal obesity but may also act as a confounder, we performed all multivariable analyses with and without controlling for total energy. We also performed the following sensitivity analyses: (1) performing the linear and logistic multivariable analyses stratified by total energy intake $(<v$. $>$ median energy intake), and (2) using a continuous measure of percentage of energy intake from ultra-processed foods (0-100\%) as the explanatory variable. Finally, as OR may overestimate prevalence ratios for common outcomes, we computed the multivariable-adjusted prevalence ratios for overweight/obesity, obesity and abdominal obesity among the full sample and stratified by sex, using Poisson regression with robust variance $^{(35)}$

Descriptive statistics and regression analyses were conducted using the NHANES sample weights in order to account for oversampling of certain populations, non-response and population coverage ${ }^{(36)}$. We used the Taylor Series Linearization variance approximation procedure to account for the complex sample design of NHANES in the variance estimation ${ }^{(36)}$. All analyses were performed using Stata/SE 14.2. Statistical significance was set to $\alpha=0 \cdot 05$.

\section{Results}

A total of 15977 adults met the study's inclusion criteria and were included in the analytical sample. The sample prevalence of $\mathrm{BMI} \geq 25 \mathrm{~kg} / \mathrm{m}^{2}, \mathrm{BMI} \geq 30 \mathrm{~kg} / \mathrm{m}^{2}$ and abdominal obesity was 69.2, $36 \cdot 1$ and $53 \cdot 0 \%$, respectively (Table 1 ). Participants consumed on average $56 \cdot 1 \%$ of their total energy intake in the form of ultra-processed foods. Ultra-processed foods provided $84.5 \%$ of total energy intake in the highest quintile of ultra-processed food consumption, compared with $25.4 \%$ of total energy intake in the lowest quintile. Compared with participants in the lowest quintile, individuals in the top quintile were younger $(39.8 v$. 43.3 years, $P<0.001)$, more likely to be female $(53.5 v .47 .7 \%$, $P=0.009)$, non-Hispanic white $(71.6 v .59 .2 \%, P<0.001)$ and have a family income $<130 \%$ of the federal poverty threshold (25.6 v. 22.7\%, $P<0.001$ ). Participants in the highest quintile had the highest mean BMI $\left(29.9 \mathrm{~kg} / \mathrm{m}^{2}, P<0.001\right)$ and WC $(100.3 \mathrm{~cm}, P<0.001)$, as well as the greatest prevalence of overweight $(71.4 \%, P=0.001)$, obesity $(41.9 \%, P<0.001)$ and abdominal obesity $(58.8 \%, P=0 \cdot 001)$. Compared with participants consuming the least ultra-processed foods (quintile 1), participants consuming the most ultra-processed foods (quintile 5) had higher average energy intake, greater relative energy contributions from carbohydrates, sugar, SFA and PUFA and lower relative intake of protein and fibre $(P=0.002$ for PUFA and $P<0.001$ for all other variables).

Table 2 shows that consumption of ultra-processed food was significantly associated with all outcomes in both age-adjusted and multivariable models adjusted for potential confounders. In multivariable analyses, the highest consumption of ultraprocessed food (quintile 5; 74.2-100\% energy) was associated with 1.61 units higher BMI (95\% CI 1.11, 2.10), $4.07 \mathrm{~cm}$ greater WC $(95 \%$ CI $2 \cdot 94,5 \cdot 19)$ and 48, 53 and $62 \%$ higher odds of having a $B M I \geq 25 \mathrm{~kg} / \mathrm{m}^{2}, \quad B M I \geq 30 \mathrm{~kg} / \mathrm{m}^{2}$ and abdominal 
Table 1. Characteristics of adults aged 20-64 years in the National Health and Nutrition Examination Survey 2005-2014 according to relative dietary contribution of ultra-processed food, $n 15977$

(Mean values and standard deviations; percentages)

\begin{tabular}{|c|c|c|c|c|c|c|c|c|c|c|c|c|c|}
\hline & \multicolumn{12}{|c|}{ Quintile of relative dietary contribution of ultra-processed food (\% of total energy) } & \multirow[b]{3}{*}{$P^{*}$} \\
\hline & \multicolumn{2}{|l|}{ All } & \multicolumn{2}{|c|}{$1(\leq 36.5 \%)$} & \multicolumn{2}{|c|}{$2(36.6-49.9 \%)$} & \multicolumn{2}{|c|}{$3(50.0-60.9 \%)$} & \multicolumn{2}{|c|}{$4(61 \cdot 0-74 \cdot 1 \%)$} & \multicolumn{2}{|c|}{$5(\geq 74 \cdot 2 \%)$} & \\
\hline & Mean & SD & Mean & SD & Mean & SD & Mean & SD & Mean & SD & Mean & SD & \\
\hline$n$ & \multicolumn{2}{|c|}{15977} & \multicolumn{2}{|c|}{3196} & \multicolumn{2}{|c|}{3195} & \multicolumn{2}{|c|}{3196} & \multicolumn{2}{|c|}{3195} & \multicolumn{2}{|c|}{3195} & \\
\hline Age (years), mean & 41.9 & 0.2 & $43 \cdot 3$ & 0.4 & $42 \cdot 6$ & 0.3 & $42 \cdot 3$ & 0.4 & 41.7 & 0.4 & $39 \cdot 8$ & 0.4 & $<0.001$ \\
\hline $\operatorname{Sex}(\%)$ & & & & & & & & & & & & & 0.009 \\
\hline Men & $49 \cdot 4$ & & $52 \cdot 3$ & & $49 \cdot 8$ & & $48 \cdot 8$ & & 49.9 & & $46 \cdot 5$ & & \\
\hline Women & $50 \cdot 6$ & & $47 \cdot 7$ & & $50 \cdot 2$ & & $51 \cdot 2$ & & $50 \cdot 1$ & & 53.5 & & \\
\hline Race (\%) & & & & & & & & & & & & & $<0.001$ \\
\hline Non-Hispanic white & 67.5 & & $59 \cdot 2$ & & 67.4 & & 67.6 & & 71.0 & & 71.6 & & \\
\hline Non-Hispanic black & $11 \cdot 3$ & & 9.7 & & $9 \cdot 3$ & & $11 \cdot 1$ & & $12 \cdot 4$ & & $13 \cdot 9$ & & \\
\hline Hispanic & $14 \cdot 2$ & & $17 \cdot 4$ & & $16 \cdot 3$ & & $16 \cdot 2$ & & $12 \cdot 1$ & & 9.5 & & \\
\hline Other & 6.9 & & $13 \cdot 7$ & & $7 \cdot 1$ & & $5 \cdot 1$ & & 4.5 & & $5 \cdot 0$ & & \\
\hline Educational attainment (\%) & & & & & & & & & & & & & $<0.001$ \\
\hline$<9$ th grade & 4.2 & & $5 \cdot 8$ & & 4.9 & & 4.4 & & 3.5 & & $2 \cdot 4$ & & \\
\hline 9th-11th grade & $10 \cdot 6$ & & $8 \cdot 8$ & & $10 \cdot 1$ & & $10 \cdot 2$ & & 11.4 & & $12 \cdot 2$ & & \\
\hline High school graduate/GED & $22 \cdot 2$ & & $18 \cdot 1$ & & $17 \cdot 5$ & & 23.1 & & $24 \cdot 1$ & & $27 \cdot 9$ & & \\
\hline Some college & $32 \cdot 7$ & & $30 \cdot 8$ & & $32 \cdot 4$ & & $30 \cdot 7$ & & 33.4 & & $35 \cdot 7$ & & \\
\hline College graduate or higher & $30 \cdot 3$ & & $36 \cdot 5$ & & $35 \cdot 1$ & & 31.5 & & $27 \cdot 7$ & & 21.9 & & \\
\hline Marital status (\%) & & & & & & & & & & & & & $<0.001$ \\
\hline Married & $55 \cdot 0$ & & $57 \cdot 7$ & & 59.5 & & 55.9 & & $55 \cdot 0$ & & $47 \cdot 3$ & & \\
\hline Separated/divorced/widowed & $15 \cdot 4$ & & 14.0 & & 14.4 & & $15 \cdot 7$ & & $15 \cdot 4$ & & $17 \cdot 3$ & & \\
\hline Not married & 29.6 & & $28 \cdot 3$ & & $26 \cdot 1$ & & 28.4 & & 29.7 & & 35.4 & & \\
\hline Family income to poverty ratio (\%) & & & & & & & & & & & & & $<0.001$ \\
\hline$<130$ & 21.8 & & $22 \cdot 7$ & & $19 \cdot 0$ & & $19 \cdot 6$ & & 21.9 & & $25 \cdot 6$ & & \\
\hline 130-349 & $32 \cdot 5$ & & 29.5 & & $31 \cdot 7$ & & 32.5 & & $33 \cdot 2$ & & $35 \cdot 4$ & & \\
\hline$\geq 350$ & $45 \cdot 7$ & & $47 \cdot 9$ & & $49 \cdot 2$ & & 48.0 & & 44.9 & & $39 \cdot 0$ & & \\
\hline $\mathrm{BMI}\left(\mathrm{kg} / \mathrm{m}^{2}\right)$ & 28.9 & 0.1 & $28 \cdot 1$ & 0.2 & 28.5 & 0.2 & 28.9 & 0.2 & $29 \cdot 1$ & 0.2 & $29 \cdot 9$ & 0.2 & $<0.001$ \\
\hline $\mathrm{BMI} \geq 25 \mathrm{~kg} / \mathrm{m}^{2}(\%)$ & $69 \cdot 2$ & & $65 \cdot 1$ & & $69 \cdot 0$ & & $69 \cdot 3$ & & 70.9 & & 71.4 & & 0.001 \\
\hline $\mathrm{BMI} \geq 30 \mathrm{~kg} / \mathrm{m}^{2}(\%)$ & $36 \cdot 1$ & & 31.3 & & 32.5 & & $36 \cdot 2$ & & 38.1 & & 41.9 & & $<0.001$ \\
\hline Waist circumference (cm) & $98 \cdot 3$ & 0.3 & $96 \cdot 4$ & 0.4 & $97 \cdot 3$ & 0.4 & 98.1 & 0.5 & 99.0 & 0.4 & $100 \cdot 3$ & 0.5 & 0.007 \\
\hline Abdominal obesity (\%) & 53.0 & & 47.5 & & $50 \cdot 2$ & & 52.7 & & 54.9 & & $58 \cdot 8$ & & $<0.001$ \\
\hline \multicolumn{14}{|l|}{ Physical activity level (incl. work) (\%) } \\
\hline Low & $40 \cdot 9$ & & $38 \cdot 0$ & & $40 \cdot 6$ & & $39 \cdot 1$ & & 41.6 & & $44 \cdot 7$ & & \\
\hline Moderate & $15 \cdot 2$ & & $16 \cdot 4$ & & $14 \cdot 0$ & & $16 \cdot 0$ & & $16 \cdot 2$ & & $13 \cdot 7$ & & \\
\hline High & $43 \cdot 9$ & & $45 \cdot 6$ & & $45 \cdot 3$ & & $44 \cdot 9$ & & $42 \cdot 2$ & & $41 \cdot 6$ & & \\
\hline Smoking status (\%) & & & & & & & & & & & & & $<0.001$ \\
\hline Never smoked & $55 \cdot 2$ & & $56 \cdot 7$ & & $57 \cdot 3$ & & $56 \cdot 1$ & & $53 \cdot 2$ & & $53 \cdot 2$ & & \\
\hline Former smoker & $21 \cdot 1$ & & $22 \cdot 2$ & & 22.5 & & 22.4 & & 21.2 & & $17 \cdot 2$ & & \\
\hline Current smoker & 23.7 & & $21 \cdot 1$ & & $20 \cdot 2$ & & 21.6 & & $25 \cdot 6$ & & $29 \cdot 6$ & & \\
\hline Total energy intake (kJ) & $8936 \cdot 2$ & 39.7 & $8455 \cdot 9$ & $98 \cdot 7$ & $9008 \cdot 6$ & $74 \cdot 1$ & $9052 \cdot 1$ & $73 \cdot 6$ & 9263.4 & 84.5 & $8848 \cdot 7$ & 92.9 & $<0.001$ \\
\hline Relative energy intake (\% energy) from & & & & & & & & & & & & & \\
\hline Unprocessed/minimally processed foods $\dagger$ & $29 \cdot 3$ & & $50 \cdot 4$ & & $37 \cdot 2$ & & 29.4 & & $22 \cdot 0$ & & $10 \cdot 3$ & & $<0.001$ \\
\hline Processed culinary ingredientsł & $4 \cdot 2$ & & $7 \cdot 0$ & & $5 \cdot 5$ & & 4.3 & & $3 \cdot 0$ & & 1.5 & & $<0.001$ \\
\hline Processed foods§ & $10 \cdot 5$ & & $17 \cdot 1$ & & $13 \cdot 7$ & & $10 \cdot 9$ & & $7 \cdot 8$ & & 3.8 & & $<0.001$ \\
\hline Ultra-processed foods\|ll & $56 \cdot 1$ & & $25 \cdot 4$ & & $43 \cdot 6$ & & 55.4 & & $67 \cdot 1$ & & 84.5 & & $<0.001$ \\
\hline Nutrient intakes (\% energy) from & & & & & & & & & & & & & \\
\hline Protein & 15.9 & & $18 \cdot 8$ & & $17 \cdot 2$ & & $16 \cdot 0$ & & 14.9 & & $13 \cdot 0$ & & $<0.001$ \\
\hline Carbohydrates & $48 \cdot 5$ & & 44.9 & & $46 \cdot 3$ & & $48 \cdot 1$ & & 50.0 & & 52.9 & & $<0.001$ \\
\hline Total sugar & 21.6 & & $17 \cdot 8$ & & 19.5 & & $21 \cdot 6$ & & $23 \cdot 3$ & & $25 \cdot 4$ & & $<0.001$ \\
\hline Total fat & 33.5 & & $32 \cdot 1$ & & $33 \cdot 7$ & & 33.8 & & 33.9 & & $33 \cdot 7$ & & $<0.001$ \\
\hline SFA & 10.9 & & $10 \cdot 1$ & & 11.0 & & $11 \cdot 0$ & & $11 \cdot 2$ & & $11 \cdot 1$ & & $<0.001$ \\
\hline MUFA & $12 \cdot 0$ & & 11.9 & & $12 \cdot 1$ & & $12 \cdot 0$ & & $12 \cdot 0$ & & 11.9 & & 0.902 \\
\hline PUFA & $7 \cdot 6$ & & $7 \cdot 2$ & & $7 \cdot 6$ & & $7 \cdot 7$ & & $7 \cdot 7$ & & $7 \cdot 7$ & & 0.002 \\
\hline Fibre (g/4184 kJ) & 8.0 & $0 \cdot 1$ & $9 \cdot 3$ & $0 \cdot 1$ & $8 \cdot 7$ & 0.1 & 8.0 & 0.1 & 7.5 & 0.1 & $6 \cdot 7$ & 0.1 & $<0.001$ \\
\hline
\end{tabular}

GED, general equivalency diploma.

* $P$ value for continuous variables is estimated through unadjusted linear regression, treating quintile as an ordinal variable, and Pearson's $x^{2}$ for categorical variables.

† Natural, packaged, cut, chilled or frozen vegetables, fruits, potatoes, cassava and other roots and tubers; white, parboiled and whole-grain rice; whole or separated maize; grains of wheat and other cereals that are dried, polished or ground as grits or flour; dried or fresh pasta made from wheat flour and water; beans; lentils, chickpeas and other legumes; dried fruits, fruit juices fresh or pasteurised without added sugar or other substances; nuts, peanuts and other oilseeds without salt or sugar; fresh and dried mushrooms; fresh and dried herbs and spices; fresh, frozen, dried beef, pork, poultry and other meat and fish; pasteurised, 'long-life' and powdered milk; fresh and dried eggs, yogurt without sugar; tea, herbal infusions, coffee and tap, spring and mineral water.

‡ Plant oils; coconut and animal fats (including butter and lard); table sugar, maple syrup (100\%), molasses and honey; and table salt.

$\S$ Canned and bottled vegetables, legumes or fruits; salted nuts or seeds; salted, smoked or cured meat or fish; canned fish or meat; cheeses.

II Instant and canned soups; reconstituted meat and fish products; ready-made sauces, gravies and dressings; French fries and other pre-made potato products such as chips; ready-to-eat and dry-mix desserts such as pudding; confectionery; sweet and savoury snack foods including granola and protein bars; sugar-sweetened or artificially sweetened beverages including soda, fruit drinks, pre-sweetened tea and coffee, energy drinks and dairy product-based drinks; flavoured and/or sweetened yogurt; industrially manufactured cakes, cookies and pies; dry cake and pancake mixes; industrially manufactured breads; processed cheese products; sweet breakfast cereals; frozen and shelf-stable plate meals; ice cream, frozen yogurt and ice pops; meatless patties and fish sticks and infant formula. 
Table 2. Age-adjusted and multivariable analyses of the association between quintile of relative dietary contribution of ultra-processed food (\% of total energy) and indicators of excess weight and abdominal obesity among adults aged 20-64 years in the National Health and Nutrition Examination Survey 2005-2014, n 15977

(Linear regression coefficients $(\beta) /$ odds ratios and $95 \%$ confidence intervals)

\begin{tabular}{|c|c|c|c|c|c|c|c|c|c|c|c|}
\hline & \multicolumn{10}{|c|}{ Quintile of relative dietary contribution of ultra-processed food (\% of total energy) } & \multirow[b]{3}{*}{$P_{\text {for trend }}$} \\
\hline & \multicolumn{2}{|c|}{$1(\leq 36.5 \%)$} & \multicolumn{2}{|c|}{$2(36 \cdot 6-49 \cdot 9 \%)$} & \multicolumn{2}{|c|}{$3(50 \cdot 0-60 \cdot 9 \%)$} & \multicolumn{2}{|c|}{$4(61 \cdot 0-74 \cdot 1 \%)$} & \multicolumn{2}{|c|}{$5(\geq 74 \cdot 2 \%)$} & \\
\hline & $\beta / \mathrm{OR}$ & $95 \% \mathrm{Cl}$ & $\beta / \mathrm{OR}$ & $95 \% \mathrm{Cl}$ & $\beta / \mathrm{OR}$ & $95 \% \mathrm{Cl}$ & $\beta / \mathrm{OR}$ & $95 \% \mathrm{Cl}$ & $\beta / \mathrm{OR}$ & $95 \% \mathrm{Cl}$ & \\
\hline \multicolumn{12}{|l|}{$\operatorname{BMI}\left(\mathrm{kg} / \mathrm{m}^{2}\right)(\beta)$} \\
\hline Age adjusted & 0.0 & Ref. & $0.48^{\star}$ & $0.02,0.93$ & $0.92^{*}$ & $0.46,1.37$ & $1 \cdot 16^{\star}$ & $0.73,1.59$ & $2 \cdot 03^{\star}$ & $1.54,2.51$ & $<0.001$ \\
\hline Multivariable $\dagger$ & 0.0 & Ref. & 0.32 & $-0.12,0.77$ & $0.66^{*}$ & $0.22,1 \cdot 10$ & $0.84^{*}$ & $0.42,1.26$ & $1.61^{*}$ & $1 \cdot 11,2 \cdot 10$ & $<0.001$ \\
\hline \multicolumn{12}{|c|}{$\mathrm{BMI} \geq 25 \mathrm{~kg} / \mathrm{m}^{2}(\mathrm{OR})$} \\
\hline Age adjusted & 1.0 & Ref. & $1.23^{\star}$ & $1.06,1.43$ & $1 \cdot 26^{\star}$ & $1.06,1.49$ & $1 \cdot 39^{\star}$ & $1.19,1.61$ & $1.51^{\star}$ & $1.29,1.77$ & $<0.001$ \\
\hline Multivariable $†$ & 1.0 & Ref. & $1 \cdot 20^{*}$ & $1.03,1.39$ & $1 \cdot 21^{*}$ & $1.02,1.43$ & $1.33^{*}$ & $1.14,1.55$ & $1.48^{\star}$ & $1.25,1.76$ & $<0.001$ \\
\hline \multicolumn{12}{|c|}{$\mathrm{BMI} \geq 30 \mathrm{~kg} / \mathrm{m}^{2}(\mathrm{OR})$} \\
\hline Age adjusted & 1.0 & Ref. & 1.07 & $0.91,1.25$ & $1 \cdot 27^{\star}$ & $1.09,1.48$ & $1 \cdot 39^{\star}$ & $1.21,1.60$ & $1.69^{\star}$ & $1.45,1.98$ & $<0.001$ \\
\hline Multivariable $†$ & 1.0 & Ref. & 1.03 & $0.87,1.20$ & $1 \cdot 19^{*}$ & $1.03,1.39$ & $1.29^{*}$ & $1.12,1.48$ & $1.53^{*}$ & $1.29,1.81$ & $<0.001$ \\
\hline \multicolumn{12}{|c|}{ Waist circumference $(\beta)$} \\
\hline Age adjusted & 0.0 & Ref. & $1 \cdot 14^{*}$ & $0.07,2.21$ & $2 \cdot 05^{\star}$ & $0.94,3.15$ & $3.07^{*}$ & $2 \cdot 14,4.01$ & $5 \cdot 00^{*}$ & $3.93,6.07$ & $<0.001$ \\
\hline Multivariable† & 0.0 & Ref. & 0.81 & $-0.23,1.86$ & $1.56^{\star}$ & $0.50,2.62$ & $2 \cdot 24^{\star}$ & $1 \cdot 31,3 \cdot 18$ & $4 \cdot 07^{\star}$ & $2 \cdot 94,5 \cdot 19$ & $<0.001$ \\
\hline \multicolumn{12}{|c|}{ Abdominal obesity (OR)‡ } \\
\hline Age adjusted & 1.0 & Ref. & $1 \cdot 15$ & $0.99,1.34$ & $1 \cdot 30^{*}$ & $1.11,1.51$ & $1.46^{\star}$ & $1.26,1.69$ & $1.87^{\star}$ & $1 \cdot 62,2 \cdot 15$ & $<0.001$ \\
\hline Multivariable† & 1.0 & Ref. & 1.08 & $0.92,1.27$ & $1 \cdot 18^{*}$ & $1.01,1.38$ & $1.32^{*}$ & $1.13,1.54$ & $1.62^{*}$ & $1.39,1.89$ & $<0.001$ \\
\hline
\end{tabular}

Ref., reference group.

${ }^{*} P<0.05$.

† Adjusted for age (years), sex, educational attainment (less than high school, high school degree/general equivalency diploma, some college, college graduate or above), race/ ethnicity (Hispanic, Non-Hispanic White, Non-Hispanic Black, other race including multi-racial), ratio of family income:poverty, marital status (married, separated/divorced/ widowed, never married) and smoking (never smoked, former smoker, current smoker) and physical activity level (low, medium, high).

‡ Defined as waist circumference $\geq 88 / 102 \mathrm{~cm}$ for women and men, respectively.

obesity, respectively $(\mathrm{OR}=1.48 ; 95 \%$ CI $1.25,1.76 ; \mathrm{OR}=1.53$; $95 \%$ CI 1.29, 1.81; and OR $=1.62 ; 95 \%$ CI 1.39, 1.89, respectively), compared with the lowest consumption (quintile 1; 0-36.5\% energy). A significant linear trend was observed for the association between quintile of ultra-processed food consumption and each outcome $(P<0 \cdot 001)$. Fig. 2 shows the multivariable-adjusted estimated margins for BMI, WC and probability of obesity and abdominal obesity according to quintile of ultra-processed food consumption at the means of all covariates. We obtained consistent results using Poisson regression. Multivariable-adjusted prevalence ratios of overweight/obesity, obesity and abdominal obesity in relation to ultra-processed food consumption are presented in the online Supplementary Table S1 of the online Supplementary material.

A significant interaction between being female and relative dietary contribution of ultra-processed food was observed for BMI $\left(F_{4,79}=4.89, P=0.002\right)$, WC $\left(F_{4,79}=3.71, P=0.008\right)$ and overweight $\left(F_{4,79}=5.35, \quad P<0.001\right)$, but not obesity and abdominal obesity. Compared with women in the lowest quintile of ultra-processed food consumption, women in the second to fifth quintile had significantly higher BMI, WC and odds of being overweight and having abdominal obesity (Table 3). Women in the third to fifth quintile had significantly higher odds of being obese compared with women in the first quintile. In contrast, associations between ultra-processed food consumption and BMI, WC and odds of obesity and abdominal obesity were only significant for men in the top compared with the lowest quintile of consumption, and no association was observed for odds of overweight. Compared with the lowest quintile of relative intake of ultra-processed food, being in the top quintile was associated with 2.37 units higher BMI among women and only 0.79 units higher BMI among men $(\beta=2.37$; $95 \%$ CI $1.58,3 \cdot 17$ and $\beta=0.79 ; 95 \%$ CI $0 \cdot 18,1 \cdot 39$, respectively). Similarly, the coefficient for WC was considerably higher for women compared with that in men in the top quintile of consumption $(\beta=5 \cdot 66 ; 95 \%$ CI 3.92, $7 \cdot 40$ and $\beta=2 \cdot 26 ; 95 \%$ CI 0.74 , $3 \cdot 77$, respectively). No significant interaction was observed for ultra-processed food consumption and age or age group.

We accounted for energy adjustment in multiple ways. Including total energy intake as a confounding variable, stratifying the sample by energy intake or treating relative dietary contribution of ultra-processed foods as a continuous variable did not meaningfully change our results (data not shown).

\section{Discussion}

In this large, nationally representative sample of US adults, higher consumption of ultra-processed food was associated with greater BMI, WC and odds of overweight, obesity and abdominal obesity. Although consumption of ultra-processed food was directly associated with excess weight and abdominal obesity in both sexes, the association was more pronounced among women.

The findings of the present study are supported by previous research suggesting an association between relative intake of ultra-processed food and excess weight ${ }^{(16-18)}$, with potential differences between sexes ${ }^{(16)}$. Louzada et al $^{(16)}$ observed a significant association between the share of total energy intake from ultra-processed food and BMI, overweight and obesity in a cross-sectional study of 30243 individuals aged $\geq 10$ years in Brazil. However, the association between ultra-processed food consumption (\% energy) and excess weight was only significant among women in sex-stratified analyses ${ }^{(16)}$. 
(a)

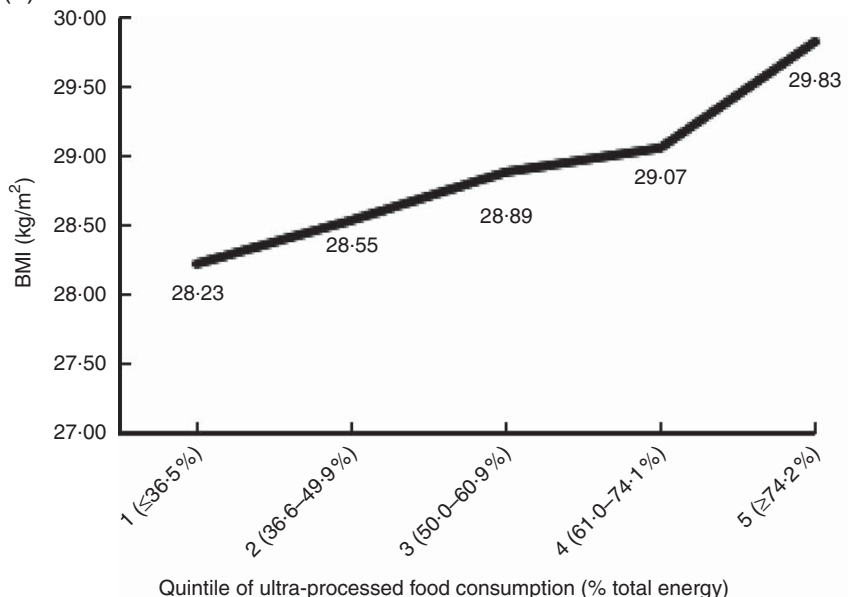

(c)

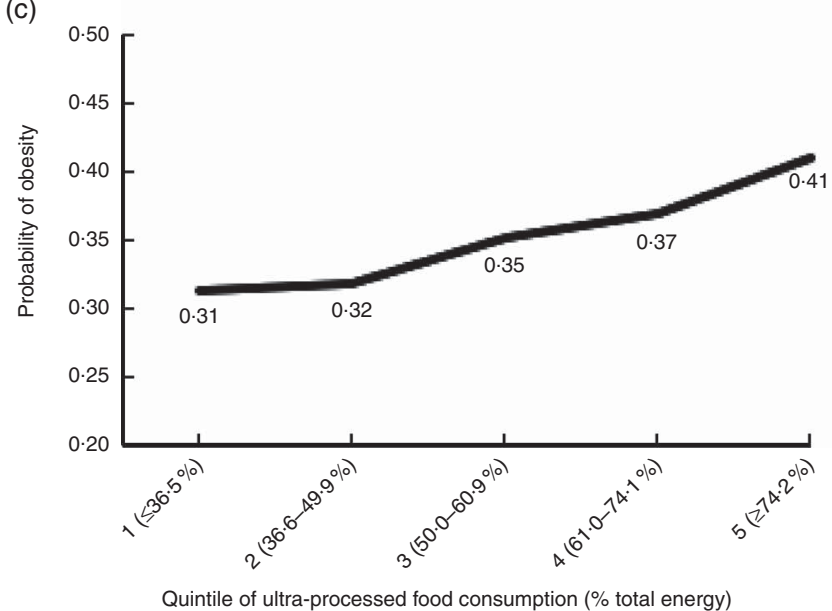

(b)

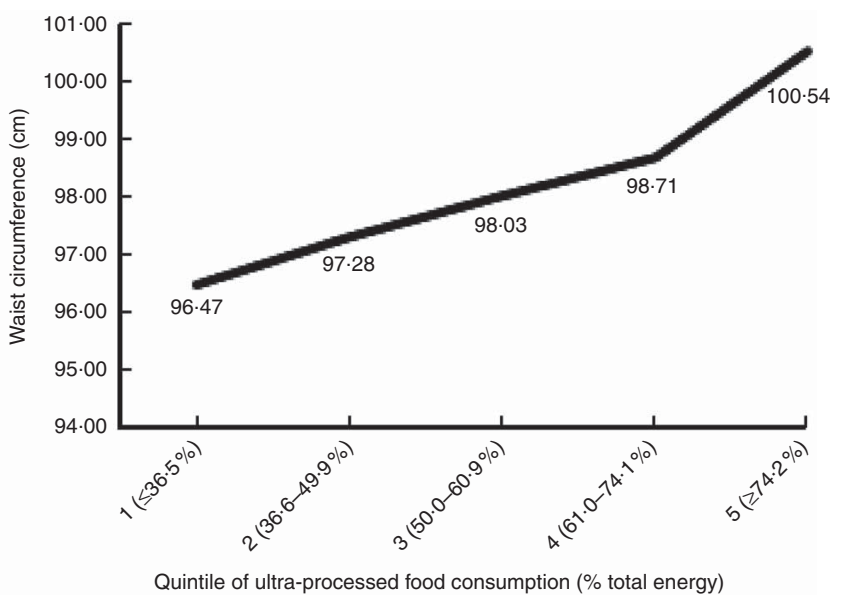

(d)

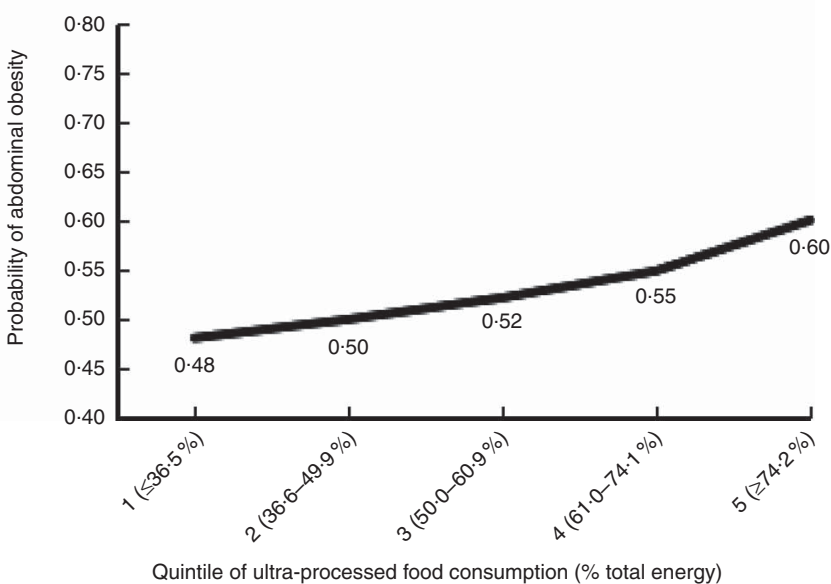

Fig. 2. Estimated mean (a) BMI, (b) waist circumference and probability of (c) obesity and (d) abdominal obesity according to quintile of relative dietary contribution of ultra-processed food (\% of total energy), based on multivariable regression models. Predicted values are adjusted for all covariates of the multivariable models and estimated at the means of all covariates.

The reasons for the observed sex differences are unclear but may be influenced by sex-related differences in food choices. In the present study, women on average, as compared with men, consumed a higher percentage of daily energy intake in the form of high-sugar ultra-processed foods such as cakes, cookies and pies $(P=0 \cdot 016)$; ice cream and frozen yogurt $(P=0 \cdot 015)$; ready-to-eat-desserts $(<0 \cdot 001)$; sweet snacks $(<0 \cdot 001)$; and milk-based sweet drinks $(<0 \cdot 001)$. Men, on the other hand, had higher relative intakes of savoury ultra-processed foods such as ultra-processed meat products $(P<0.001)$, potato products such as chips and French fries $(P=0.038)$ and frozen pizza $(P=0 \cdot 01)$, but also regular soda $(P<0 \cdot 001)$. As a result, women had higher average relative intakes of carbohydrates (49.6 $\mathrm{v}$. $47.5 \%$ of total energy; $P<0.001)$ and total sugars $(22.4 v .20 .9 \%$ of total energy, $P<0 \cdot 001$ ) (results not shown).

An accumulating body of evidence indicates that foods with high glycaemic index and glycaemic load may have more harmful metabolic effects and influence adiposity to a greater extent among women than among men ${ }^{(37-39)}$. Therefore, it can be hypothesised that a higher intake of sugary foods and refined carbohydrates, in combination with a greater sensitivity to the hyper-glycaemic effects of these foods, is contributing to the stronger association between ultra-processed foods and excess weight and abdominal obesity among women. As we observed an association between ultra-processed foods and BMI and WC only among the highest quintile of ultra-processed food consumption among men (consuming $\geq 74.2 \%$ energy from ultra-processed foods), it can be speculated that higher consumption levels are necessary to influence body weight and abdominal obesity among men than among women.

Ultra-processed foods may contribute to weight gain through their nutritional profile, by displacing low-energy nutrientdense unprocessed and minimally processed foods from the diet, and by promoting poor dietary habits ${ }^{(1-3)}$. The convenience, omnipresence, affordability, large portion sizes and aggressive marketing of ultra-processed food promotes constant snacking and involuntary over-eating, and may dislocate less processed, more nutritious foods from the diet ${ }^{(1-3)}$. Ultraprocessed products may also facilitate over-eating as they are less satiating than less processed foods ${ }^{(40)}$. The high-intensity flavouring resulting from high levels of fat, salt, sugar and artificial flavourings make ultra-processed products extremely 
Table 3. Sex-stratified multivariable analyses of the association between quintile of relative dietary contribution of ultra-processed food (\% of total energy) and indicators of excess weight and abdominal obesity among adults aged 20-64 years in the National Health and Nutrition Examination Survey 2005$2014, n 15977$

(Linear regression coefficients $(\beta) /$ odds ratios and $95 \%$ confidence intervals)

\begin{tabular}{|c|c|c|c|c|c|c|c|c|c|c|c|}
\hline & \multicolumn{10}{|c|}{ Quintile of relative dietary contribution of ultra-processed food (\% of total energy) } & \multirow[b]{3}{*}{$P_{\text {for trend }}$} \\
\hline & \multicolumn{2}{|c|}{$1(\leq 36.5 \%)$} & \multicolumn{2}{|c|}{$2(36 \cdot 6-49 \cdot 9 \%)$} & \multicolumn{2}{|c|}{$3(50 \cdot 0-60 \cdot 9 \%)$} & \multicolumn{2}{|c|}{$4(61 \cdot 0-74 \cdot 1 \%)$} & \multicolumn{2}{|c|}{$5(\geq 74 \cdot 2 \%)$} & \\
\hline & $\beta / \mathrm{OR}$ & $95 \% \mathrm{Cl}$ & $\beta / O R$ & $95 \% \mathrm{Cl}$ & $\beta / O R$ & $95 \% \mathrm{Cl}$ & $\beta / \mathrm{OR}$ & $95 \% \mathrm{Cl}$ & $\beta / O R$ & $95 \% \mathrm{Cl}$ & \\
\hline \multicolumn{12}{|l|}{$\operatorname{BMI}\left(\mathrm{kg} / \mathrm{m}^{2}\right)(\beta) \dagger$} \\
\hline Women $(n$ 8115) & 0.0 & Ref. & $0.74^{*}$ & $0.02,1.46$ & $1.38^{*}$ & $0.71,2.04$ & $1.53^{*}$ & $0.85,2 \cdot 22$ & $2 \cdot 37^{\star}$ & $1.58,3.17$ & $<0.001$ \\
\hline Men ( $n$ 7862) & 0.0 & Ref. & -0.01 & $-0.61,0.37$ & -0.01 & $-0.58,0.56$ & $0 \cdot 18$ & $-0.31,0.66$ & $0.79^{*}$ & $0.18,1.39$ & 0.005 \\
\hline \multicolumn{12}{|c|}{$\mathrm{BMI} \geq 25 \mathrm{~kg} / \mathrm{m}^{2}(\mathrm{OR}) \dagger$} \\
\hline Women (n 8115) & 1.0 & Ref. & $1.42^{\star}$ & $1 \cdot 13,1.78$ & $1.51^{\star}$ & $1.24,1.85$ & $1.65^{\star}$ & $1.32,2.05$ & $1 \cdot 88^{\star}$ & $1.47,2.39$ & $<0.001$ \\
\hline Men $(n 7862)$ & 1.0 & Ref. & 0.97 & $0.79,1.19$ & 0.94 & $0.73,1.20$ & 1.04 & $0.85,1.28$ & 1.12 & $0.88,1.42$ & 0.276 \\
\hline \multicolumn{12}{|l|}{$\mathrm{BMl} \geq 30 \mathrm{~kg} / \mathrm{m}^{2}(\mathrm{OR}) \dagger$} \\
\hline Women $(n$ 8115) & 1.0 & Ref. & 1.18 & $0.93,1.50$ & $1.44^{\star}$ & $1 \cdot 16,1 \cdot 80$ & $1.48^{*}$ & $1.22,1.79$ & $1 \cdot 79^{\star}$ & $1 \cdot 37,2 \cdot 34$ & $<0.001$ \\
\hline Men $(n 7862)$ & 1.0 & Ref. & 0.90 & $0.74,1.09$ & 1.01 & $0.82,1.25$ & 1.15 & $0.94,1.41$ & $1.33^{*}$ & $1.04,1.69$ & 0.003 \\
\hline \multicolumn{12}{|c|}{ Waist circumference $(\beta) \dagger$} \\
\hline Women ( $n$ 8115) & 0.0 & Ref. & $1.93^{*}$ & $0.29,3.57$ & $2.98^{*}$ & $1.50,4.47$ & $3.41^{*}$ & $1.94,4.89$ & $5 \cdot 66^{*}$ & $3.92,7.40$ & $<0.001$ \\
\hline Men $(n$ 7862) & 0.0 & Ref. & -0.39 & $-1.56,0.78$ & 0.09 & $-1.41,1.58$ & 0.97 & $-0.36,2.30$ & $2 \cdot 26^{\star}$ & $0.74,3.77$ & $<0.001$ \\
\hline \multicolumn{12}{|c|}{ Abdominal obesity (OR)†‡ } \\
\hline Women ( $n$ 8115) & 1.0 & Ref. & $1 \cdot 28^{*}$ & $1.02,1.59$ & $1.33^{*}$ & $1.07,1.66$ & $1.51^{\star}$ & $1.18,1.93$ & $1 \cdot 80^{\star}$ & $1 \cdot 44,2 \cdot 24$ & $<0.001$ \\
\hline Men $(n 7862)$ & 1.0 & Ref. & 0.91 & $0.72,1.14$ & 1.05 & $0.84,1.31$ & $1 \cdot 16$ & $0.93,1.44$ & $1.47^{\star}$ & $1.20,1.80$ & $<0.001$ \\
\hline
\end{tabular}

Ref., reference group.

* $P<0.05$.

† Adjusted for age (years), sex, educational attainment (less than high school, high school degree/general equivalency diploma, some college, college graduate or above), race/ ethnicity (Hispanic, Non-Hispanic White, Non-Hispanic Black, other race including multi-racial), ratio of family income to poverty, marital status (married, separated/divorced/ widowed, never married) and smoking (never smoked, former smoker, current smoker) and physical activity level (low, medium, high).

‡ Defined as waist circumference $\geq 88 / 102 \mathrm{~cm}$ for women and men, respectively.

palatable, for which endogenous satiety mechanisms may be superseded ${ }^{(31)}$. In addition, ultra-processed foods are, on average, more energy-dense than unprocessed and minimally processed foods and culinary preparations based on minimally processed foods $^{(1,2)}$. As human satiety mechanisms are more sensitive to volume than energetic content, foods with higher energy density may facilitate excessive energy intakes ${ }^{(41)}$.

Beyond nutrient composition, greater deconstruction of the original food matrix, as occurs in processing, has been associated with lower satiety and higher glycaemic response ${ }^{(40)}$. Ultra-processed foods were found to be significantly less satiating and more hyper-glycaemic than minimally processed foods in an experimental study of ninety-eight food items ${ }^{(40)}$. Finally, food structure and fibre content of the diet influence the gut microbiomic profile and integrity, with potential important implications for body weight and adiposity ${ }^{(42)}$. The gut microbiota is altered in obesity, and evidence from both human and animal studies suggests that the gut microbiota effects energy harvest, energy homoeostasis and lipid accumulation of the host, although the biological mechanisms are not completely understood $^{(43)}$.

To our knowledge, this is the first study to examine the association between consumption of ultra-processed food, defined per NOVA, and excess weight in a national population of the USA. The use of NOVA is a key strength of the study as it enabled food-processing level to be determined according to standardised and objective criteria. NOVA has been recognised as a valid tool for public health and nutrition research and policy by the FAO of the United Nations ${ }^{(44)}$ and the Pan American Health Organization ${ }^{(45)}$. The high-quality design and methodology of NHANES is another important strength of the present study. Importantly, dietary intakes are assessed with a sufficient level of detail to enable determination of processing level. The dietary data also allowed for the disaggregation of dishes into their constituents and classification of the underlying ingredients, which enabled the calculation of more precise estimates of intakes of each NOVA group and reduced the risk of misclassification. Finally, as NHANES is nationally representative, the external validity of the findings is high.

Some limitations should be noted. Owing to the crosssectional nature of the data, temporality and causality cannot be established and reverse causality cannot be excluded. However, consumption of ultra-processed foods was significantly associated with incident overweight and obesity in the prospective Spanish SUN study, thereby showing a linkage between the consumption of ultra-processed foods and subsequent, excess weight ${ }^{(17)}$. As is common in nutritional epidemiologic studies, dietary assessment by $24-\mathrm{h}$ recall provides an imperfect measure of diet, and foods consumed on the assessment day may not be representative of usual diet. However, the AMPM is a validated method for dietary data collection that has been shown to reduce bias in dietary data collection $^{(26,27)}$.

Using relative consumption as the exposure variable attenuates the effect of total energy intake and mitigates bias introduced by misreporting as long as individuals misreport energy intake from all foods to the same extent. Nevertheless, research suggests that individuals may be more likely to under-report foods considered unhealthy, including ultra-processed foods such as confectionery, pastries and French fries, compared with minimally processed foods such as vegetables ${ }^{(46)}$. However, if obese individuals are more likely to under-report consumption 
of ultra-processed foods, the association would be biased towards the null. Finally, misclassification of foods cannot be excluded, as it was not always possible to determine NOVA group with certainty owing to insufficient information. We used a conservative approach, such that the lower level of processing was always assigned in case of uncertainty. Potential bias arising from misclassification would therefore lead to underestimation of ultra-processed food consumption and would not necessarily affect the association between ultra-processed foods and excess weight. Despite potential underestimation of the exposure, we observed a strong association between ultraprocessed food consumption and excess weight and abdominal obesity, which is biologically plausible and consistent with the existing literature.

To date, little attention has been paid to the potential role of food processing in excess weight and adiposity. In recent years, nutrition research and dietary guidelines have increasingly focused on dietary patterns and food groups such as fruits, vegetables and whole grains, rather than just nutrients. Nevertheless, except for processed meat, the form in which foods are consumed and how they have been processed is generally not addressed $^{(47)}$. In consequence, individual foods with diverse characteristics are often categorised within the same exposure group in nutritional studies; for example both brown rice and sugary and artificially flavoured whole-wheat breakfast cereals may count towards whole-grain consumption. This approach probably limits the ability to differentiate foods with beneficial and hazardous health effects owing to processing and may lead to inconsistent and contradictory findings ${ }^{(47)}$. Similarly, recommendations to consume certain food groups without specifying processing level may not provide sufficient guidance to allow consumers to make healthful choices. For example, recommendations to consume more vegetables do not distinguish between fresh vegetables and instant vegetable soups, which are generally high in Na. Conversely, the classification of foods according to level of processing enables the identification of food groups with similar characteristics, nutritional quality and potential health effects. Importantly, NOVA takes into account that both the nutritional (e.g. chemical additives, macronutrient and micronutrient content) and non-nutritional attributes (e.g. mode of consumption, marketing) of foods may affect eating behaviour, nutritional quality and health outcomes.

\section{Conclusion}

Our findings suggest that ultra-processed foods may contribute to the high rates of excess weight and abdominal obesity in the USA. This study makes an important contribution to the literature as the first study to investigate this association in a US setting, confirming findings from Brazil, France and Spain. Given the unprecedented rates of obesity worldwide ${ }^{(48)}$, it is crucial to further elucidate the role of ultra-processed foods in the development of excess weight and adiposity. Moreover, studies are needed to improve our understanding of the biological, social or cultural drivers of potential sex differences. Enhanced knowledge regarding the influence of food-processing level on excess weight has potential implications for food production businesses, one of the fastestgrowing industries. These results, if furthermore confirmed in longitudinal studies, raise the question of whether specific recommendations to distinguish ultra-processed from moderately and minimally processed foods should be included as an extension of the food-based US dietary recommendations. In clinical practice level, a better comprehension of the associations between food processing and excess weight may aid in individual dietary guidance and weight management strategies.

\section{Acknowledgements}

This research received no specific grant from any funding agency or from commercial or not-for-profit sectors. While the present study was unfunded, the initial work of C. A. M. and E. M.-S. to classify the NHANES dietary data according to NOVA was supported by the Sao Paulo Research Foundation (FAPESP) grant number 2015/14900-9.

F. J. and V. W. C. developed the overall research plan and designed the statistical analyses within the existing data set; F. J. performed the statistical analyses and took the lead in writing the manuscript; E. M.-S. and F. J. classified the dietary data into the primary exposure variable; E. M.-S. aided in the statistical analyses and interpretation of the data and revised the manuscript for critical intellectual content; N. P. provided expertise regarding statistical analyses as it pertains to nutritional epidemiology and revised the manuscript for important intellectual content; C. A. M. developed and defined the NOVA classification that was used in the present study, provided guidance regarding the classification of the dietary data and revised the article for critical intellectual content; V. W. C. participated in the analysis and interpretation of data, revised the manuscript for important intellectual content, and oversaw the entire study. All authors approved the final manuscript and take full responsibility for the final content.

The authors declare that there are no conflicts of interest.

\section{Supplementary material}

For supplementary material/s referred to in this article, please visit https://doi.org/10.1017/S0007114518001046

\section{References}

1. Moubarac JC, Parra DC, Cannon G, et al. (2014) Food classification systems based on food processing: significance and implications for policies and actions: a systematic literature review and assessment. Curr Obes Rep 3, 256-272.

2. Monteiro CA, Moubarac JC, Cannon G, et al. (2013) Ultraprocessed products are becoming dominant in the global food system. Obes Rev 14, Suppl. 2, 21-28.

3. Moubarac JC, Martins AP, Claro RM, et al. (2013) Consumption of ultra-processed foods and likely impact on human health. Evidence from Canada. Public Health Nutr 16, 2240-2248.

4. Poti JM, Mendez MA, Ng SW, et al. (2015) Is the degree of food processing and convenience linked with the nutritional quality of foods purchased by US households? Am J Clin Nutr 101, 1251-1262.

5. Martinez Steele E, Baraldi LG, Louzada ML, et al. (2016) Ultraprocessed foods and added sugars in the US diet: evidence from a nationally representative cross-sectional study. $B M J$ Open 6, e009892. 
6. Eicher-Miller HA, Fulgoni VL 3rd \& Keast DR (2012) Contributions of processed foods to dietary intake in the US from 2003-2008: a report of the Food and Nutrition Science Solutions Joint Task Force of the Academy of Nutrition and Dietetics, American Society for Nutrition, Institute of Food Technologists, and International Food Information Council. J Nutr 142, 2065S-2072S.

7. Eicher-Miller HA, Fulgoni VL \& Keast DR (2015) Processed food contributions to energy and nutrient intake differ among US children by race/ethnicity. Nutrients $\mathbf{7}$, 10076-10088.

8. Martinez Steele E, Popkin BM, Swinburn B, et al. (2017) The share of ultra-processed foods and the overall nutritional quality of diets in the US: evidence from a nationally representative cross-sectional study. Popul Health Metr 15, 6.

9. Moubarac JC, Batal M, Louzada ML, et al. (2017) Consumption of ultra-processed foods predicts diet quality in Canada. Appetite 108, 512-520.

10. Adams J \& White M (2015) Characterisation of UK diets according to degree of food processing and associations with socio-demographics and obesity: cross-sectional analysis of UK National Diet and Nutrition Survey (2008-12). Int J Behav Nutr Phys Act 12, 160.

11. Louzada ML, Martins AP, Canella DS, et al. (2015) Impact of ultra-processed foods on micronutrient content in the Brazilian diet. Rev Saude Publica 49, 45.

12. Julia C, Martinez L, Allès B, et al. (2018) Contribution of ultraprocessed foods in the diet of adults from the French NutriNetSanté study. Public Health Nutr 21, 27-37.

13. Batal M, Johnson-Down L, Moubarac JC, et al. (2018) Quantifying associations of the dietary share of ultra-processed foods with overall diet quality in First Nations peoples in the Canadian provinces of British Columbia, Alberta, Manitoba and Ontario. Public Health Nutr 21, 103-113.

14. Cediel G, Reyes M, da Costa Louzada ML, et al. (2018) Ultra-processed foods and added sugars in the Chilean diet (2010). Public Health Nutr 21, 125-133.

15. Lustig RH (2017) Processed food-an experiment that failed. JAMA Pediatr 171, 212-214.

16. Louzada ML, Baraldi LG, Steele EM, et al. (2015) Consumption of ultra-processed foods and obesity in Brazilian adolescents and adults. Prev Med 81, 9-15.

17. Mendonca RD, Pimenta AM, Gea A, et al. (2016) Ultraprocessed food consumption and risk of overweight and obesity: the University of Navarra Follow-Up (SUN) cohort study. Am J Clin Nutr 104, 1433-1440.

18. Canella DS, Levy RB, Martins AP, et al. (2014) Ultra-processed food products and obesity in Brazilian households (20082009). PLOS ONE 9, e92752.

19. Pereira MA, Kartashov AI, Ebbeling CB, et al. (2005) Fast-food habits, weight gain, and insulin resistance (the CARDIA study): 15-year prospective analysis. Lancet 365, 36-42.

20. Mozaffarian D, Hao T, Rimm EB, et al. (2011) Changes in diet and lifestyle and long-term weight gain in women and men. $N$ Engl J Med 364, 2392-2404.

21. Zipf G, Chiappa M \& Porter K. (2013) National Health and Nutrition Examination Survey: plan of operations, 1999-2010. Vital Health Stat 56, 1.

22. Centers for Disease Control and Prevention (2011) National Health and Nutrition Examination Survey (NHANES) Anthropometry Procedures Manual. Hyattsville, MD: CDC, National Center for Health Statistics (NCHS).

23. Centers for Disease Control and Prevention (2009) National Health and Nutrition Examination Survey (NHANES) Anthropometry Procedures Manual. Hyattsville, MD: CDC, National Center for Health Statistics (NCHS).
24. Centers for Disease Control and Prevention (2007) National Health and Nutrition Examination Survey (NHANES) Anthropometry Procedures Manual. Hyattsville, MD: CDC. National Center for Health Statistics (NCHS).

25. Centers for Disease Control and Prevention (2005) National Health and Nutrition Examination Survey Anthropometry and Physical Activity Monitor Procedures Manual. Hyattsville, MD: CDC, National Center for Health Statistics (NCHS).

26. Moshfegh AJ, Rhodes DG, Baer DJ, et al. (2008) The US Department of Agriculture Automated Multiple-Pass Method reduces bias in the collection of energy intakes. Am J Clin Nutr 88, 324-332.

27. Blanton CA, Moshfegh AJ, Baer DJ, et al. (2006) The USDA Automated Multiple-Pass Method accurately estimates group total energy and nutrient intake. J Nutr 136, 2594-2599.

28. Raper N, Perloff B, Ingwersen L, et al. (2004) An overview of USDA's Dietary Intake Data System. J Food Comp Anal 17, 545-555.

29. Centers for Disease Control and Prevention (2013) National Health and Nutrition Examination Survey: Analytic Guidelines, 2011-2012. Hyattsville, MD: Division of Health and Nutrition Examination Surveys, National Center for Health Statistics, CDC.

30. Monteiro CA (2009) Nutrition and health. The issue is not food, nor nutrients, so much as processing. Public Health Nutr 12, 729-731.

31. Ludwig DS (2011) Technology, diet, and the burden of chronic disease. JAMA 305, 1352-1353.

32. World Health Organization (2016) BMI classification. http:// apps.who.int/bmi/index.jsp?introPage=intro_3.html (accessed April 2016).

33. National Institutes of Health/National Heart, Lung, and Blood Institute (1998) Clinical guidelines on the identification, evaluation, and treatment of overweight and obesity in adults - the evidence report. Obes Res 6, Suppl. 2, 51S-209S.

34. United States Department of Health and Human Services (2008) Physical Activity Guidelines for Americans. Washington, DC: USDHHS.

35. Barros AJ \& Hirakata VN (2003) Alternatives for logistic regression in cross-sectional studies: an empirical comparison of models that directly estimate the prevalence ratio. $B M C$ Med Res Methodol 3, 21.

36. Rothwell CJ, Madans JH \& Porter K (2013) National Health and Nutrition Examination Survey: analytic guidelines, 1999-2010. Vital Health Stat 2, 161.

37. Mirrahimi A, Chiavaroli L, Srichaikul K, et al. (2014) The role of glycemic index and glycemic load in cardiovascular disease and its risk factors: a review of the recent literature. Curr Atheroscler Rep 16, 381.

38. Fan J, Song Y, Wang Y, et al. (2012) Dietary glycemic index, glycemic load, and risk of coronary heart disease, stroke, and stroke mortality: a systematic review with meta-analysis. PLOS ONE 7, e52182.

39. Youn S, Woo HD, Cho YA, et al. (2012) Association between dietary carbohydrate, glycemic index, glycemic load, and the prevalence of obesity in Korean men and women. Nutr Res 32, 153-159.

40. Fardet A (2016) Minimally processed foods are more satiating and less hyperglycemic than ultra-processed foods: a preliminary study with 98 ready-to-eat foods. Food Funct 7, 2338-2346.

41. Rolls BJ (2009) The relationship between dietary energy density and energy intake. Physiol Behav 97, 609-615.

42. Wahlqvist ML (2016) Food structure is critical for optimal health. Food Funct 7, 1245-1250. 
43. Boulange CL, Neves AL, Chilloux J, et al. (2016) Impact of the gut microbiota on inflammation, obesity, and metabolic disease. Genome Med 8, 42.

44. Food and Agriculture Organization (2015) Guidelines on the Collection of Information on Food Processing Through Food Consumption Surveys. Rome: FAO.

45. Pan American Health Organization (2015) Ultra-Processed Food and Drink Products in Latin America: Trends, Impact on Obesity, Policy Implications. Washington, DC: PAHO.

46. Lafay L, Mennen L, Basdevant A, et al. (2000) Does energy intake underreporting involve all kinds of food or only specific food items? Results from the Fleurbaix Laventie
Ville Sante (FLVS) study. Int J Obes Relat Metab Disord 24 1500-1506.

47. Fardet A, Rock E, Bassama J, et al. (2015) Current food classifications in epidemiological studies do not enable solid nutritional recommendations for preventing diet-related chronic diseases: the impact of food processing. Adv Nutr $\mathbf{6}$, 629-638.

48. Ng M, Fleming T, Robinson M, et al. (2014) Global, regional, and national prevalence of overweight and obesity in children and adults during 1980-2013: a systematic analysis for the Global Burden of Disease Study 2013. Lancet 384, $766-781$. 